\title{
The sutureless excimer laser assisted non-occlusive anastomosis (SELANA); a feasibility study in a pressurized cadaver model
}

\author{
Tristan P. C. van Doormaal • Albert van der Zwan • Emad Aboud • \\ Jan Willem Berkelbach van der Sprenkel • Cornelius A. F. Tulleken • Ali F. Krisht • \\ Luca Regli
}

Received: 27 March 2010 /Accepted: 10 June 2010 /Published online: 30 June 2010

(C) The Author(s) 2010. This article is published with open access at Springerlink.com

\begin{abstract}
Objective To compare intracranial feasibility of the conventional Excimer laser assisted non-occlusive anastomosis (ELANA) with the new experimental sutureless ELANA (SELANA).

Methods Four pressurized human cadaver heads were bilaterally trepanated, using a combined pterional/pretemporal/ transcavernous approach. In each head, seven ELANA anastomoses and seven contralateral SELANA anastomoses were constructed on (1) the proximal PCA/basilar artery (P1 segment/basilar artery; BA), (2) the distal posterior cerebral artery (PCA, P2 segment), (3) the supraclinoidal internal carotid artery (ICA), (4) the ICA bifurcation, (5) the proximal anterior cerebral artery (ACA, A1 segment), (6) the proximal middle cerebral artery (MCA, M1 segment), and (7) the distal MCA (M2 segment).

Results In total, 26 of 28 ELANA anastomoses (93\%) and 22 of 28 SELANA anastomoses (79\%) could be completed. Two ELANA anastomoses on the BA could not be finished because of limited space. Six SELANA anastomoses could not be attached because the applicator
\end{abstract}

T. P. C. van Doormaal $(\bowtie) \cdot A$. van der Zwan •

J. W. Berkelbach van der Sprenkel • C. A. F. Tulleken · L. Regli

Department of Neurosurgery,

Rudolf Magnus Institute for Neuroscience,

University Medical Center Utrecht,

Heidelberglaan 100, PO box 85500, 3508 GA Utrecht,

The Netherlands

e-mail: T.vandoormaal@gmail.com

E. Aboud · A. F. Krisht

Department of Neurosurgery,

University of Arkansas for Medical Sciences (UAMS),

Little Rock, AR, USA did not facilitate an angulated anastomosis spot. Of the remaining anastomoses, more ELANA (eight) than SELANA (two) anastomoses could not be realized without manipulation of surrounding structures. The SELANA anastomoses were completed significantly faster than the ELANA, mean difference ranging from $11 \mathrm{~min}$ on the M2 to $107 \mathrm{~min}$ on the P1/BA.

Conclusion This comparative study shows potential advantages of the SELANA anastomosis over the ELANA anastomosis because during application, it causes less manipulation of surrounding structures while it is faster and easier. Further preclinical research should be performed in order to improve SELANA feasibility on angulated anastomosis spots and to assess long-term SELANA patency and endothelialization.

Keywords Cerebral revascularization · Bypass · ELANA

\section{Introduction}

The Excimer laser assisted non-occlusive anastomosis (ELANA) technique allows the intracranial construction of an end-to-side anastomosis between a donor vessel and a recipient cerebral artery without the need to temporarily occlude the recipient artery. It therefore eliminates ischemic risk for the patient during anastomosis construction. The ELANA technique is CE approved in Europe and is currently reviewed in a prospective trail for Food and Drug Administration (FDA) approval.

The ELANA technique involves eight intracranial microsutures. This requires a high level of microsurgical skill. Every suture is accompanied by potential complications like breaking of the suture, rupturing the recipient artery 
wall, or damage of surrounding structures. If the sutures are asymmetrically applied, this can cause asymmetry of the recipient vessel wall tension within the ring. This reduces the likelihood of successful ELANA lasing. The difficulty and the risk of complications increase when the recipient artery is located deeply and when the working channel is narrow.

To overcome these difficulties, we developed a new version of the ELANA technique, the so-called sutureless ELANA (SELANA). We present a feasibility study of this technique in a pressurized human cadaver head model.

\section{Methods}

Cadaver model

We operated on four pressurized cadaveric heads at the 'Yașargil Microneurosurgery Laboratory' of the department of neurosurgery at the University of Arkansas for Medical Sciences, Little Rock.

The cadaver model preparation was performed similar to methods previously described. [1] In short, after decapitation of a formalin fixed cadaver at approximately $\mathrm{C} 6 / \mathrm{C} 7$, the common carotid arteries (CA) and vertebral arteries (VA) were exposed and cannulated. In addition, an 8-10gage tube was intradurally inserted left and right from the spinal canal and advanced to reach the intracranial subarachnoid space. Subsequently, the canal was plugged with bone wax. Tap water was used to repeatedly irrigate and flush the vessels and the subarachnoid space to remove clots, tissue debris, and formalin fixative. Leaks from arteries or veins on the sectioned surface of the neck were sealed either using ligation or coagulation. Subsequently, artificial blood was made with a mixture of red poster paint and water. Serum bags with artificial blood were attached to the CA's and VA's. Pressure bags were put over the serum bags. The arterial pressure bags were connected to a balloon pump (Datascope ${ }^{\circledR} 90 \mathrm{~T}$ ) providing pulsating pressure. We selected a rate of 60 pulses per minute, a baseline pressure of $80 \mathrm{~mm} \mathrm{Hg}$ and a 'systolic' peak pressure of $110 \mathrm{mmHg}$.

On each of the four cadaver heads, a bilateral combined pterional/pretemporal/transcavernous approach was performed as earlier described. [2, 3, 5, 11] In short, a standard pterional skin incision was made that extended below the zygoma, just anterior to the tragus. The skin flap was separated from the temporal fascia down to the fat pad over the zygoma. The temporal muscle was cut anteriorly and superiorly and was retracted inferiorly. The bone flap included the anterior temporal squama, and extended down to the temporal floor anteriorly. The pterion and the sphenoidal wings were drilled to expose the dura over the anterior temporal pole. The dura was cut parallel to the sylvian fissure, the medial cisterns were opened, the Sylvian fissure was opened laterally to medially and Liliequist's membrane was opened. Subsequently, the anterior clinoid process was removed. The roof of the cavernous sinus was incised just medially to the third nerve. Since the venous system was not pressurized, no leak occurred. The incision was progressively enlarged posteriorly until the posterior clinoid process was encountered which was also removed. This exposure facilitated a good view on all arteries on which we planned an anastomosis, including the MCA after the bifurcation (M2), the proximal MCA (M1), the ICA bifurcation, the proximal ICA, the proximal ACA (A1), the PCA after the posterior communicating artery (P2) and the proximal PCA (P1/basilar artery; BA).

\section{Anastomoses}

Before starting this study, application of ELANA and SELANA anastomoses was trained on the ELANA practice model [7] to minimize the influence of a learning curve. All anastomoses were made by the first author (TPCVD).

For all conventional ELANA anastomoses, we used thoracic rabbit aorta's as bypass graft. These were harvested in a consumption slaughterhouse from rabbits of 3-4 kg. All side branches were occluded with microsutures.

To make a conventional ELANA anastomosis (Fig. 1a), we first attached a $2.8 \mathrm{~mm}$ ring to the graft with eight microsutures. Subsequently, we attached the graft including the ring end to the side of the recipient artery, also using eight microsutures. Then, the anastomosis was lased open, using the ELANA catheter 2.0 (Elana b.v. ${ }^{\circledR}$, Utrecht, the Netherlands) and a full thickness piece of recipient artery wall (the flap) was retrieved at the suction portion of the catheter (Fig. 1b). We put an aneurysm clip on the vein graft and checked the anastomosis for patency and leakage.

To make a SELANA anastomosis, a newly designed SELANA ring was used (Fig. 2a). This ring was equipped with two pins. The SELANA ring was first mounted on the end of a thin-walled polytetrafluoroethylene (ePTFE) graft with a diameter of $3 \mathrm{~mm}$ (W. L. Gore \& Associates Inc, Flagstaff, AZ, USA) by our technician in the University Medical Center, Utrecht, The Netherlands (Fig. 2b). The SELANA was then mounted on the tip of a specially designed SELANA applicator (Fig. 2c, d). The SELANA was slid on to the recipient artery wall using the special form of the two pins (Fig. 3). First, the longitudinal parts of the two pins were simultaneously inserted in the vessel wall. When the resistance of the circumferential part of the pins was felt, the pins were aimed inside out again. Then the ring was gently pushed further in this direction, and the vessel wall 'clicked' over the circumferential part of the 


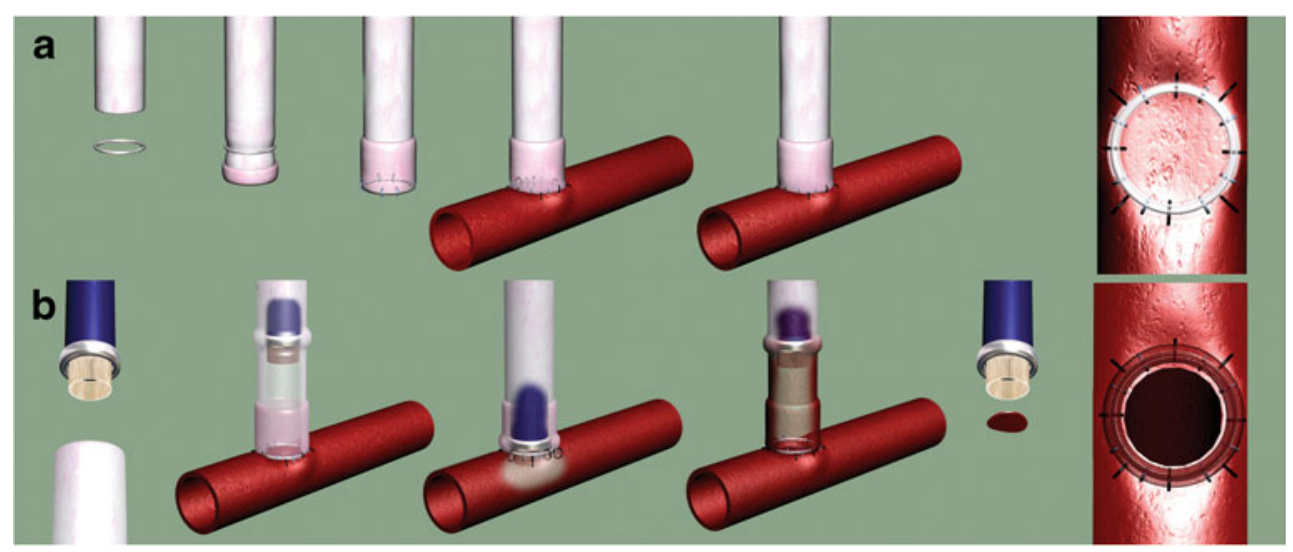

Fig. 1 Conventional ELANA anastomosis. a First, the ring is attached to the donor vein with eight microsutures (this step has not been included in the comparison of time intervals as it is performed ouside the head). Subsequently, the ring plus vein is attached to the recipient artery with eight microsutures, four of which are placed trough the ring and four outside the ring. The recipient artery is never

pins. In this way, only the circumferential part of each pin was exposed in the lumen of the artery (Fig. 3). The longitudinal parts of the pins were situated extraluminally on the arterial wall. Until this point, conversion was possible because the SELANA device can be removed again from the artery by pulling the device backward while holding the artery. This does not result in recipient vessel wall rupture, and the eventual leakage from the four perforation points is self-limiting. Subsequently, the anastomosis was sealed by a circumferential layer of Bioglue ${ }^{\circledR}$ (Cryolife ${ }^{\circledR}$ Inc., Kennesaw, GA, USA) surrounding the ring on the abluminal part. The anastomosis was lased open with the ELANA $2.0{ }^{\circledR}$ catheter, identically to the conventional ELANA anastomosis (Fig. 1b). temporary occluded during this attachment. b The ELANA catheter $2.0^{\circledR}$ is inserted into the donor vein to laser out a disk of recipient artery wall from the anastomosis (the flap), resulting in a nonocclusive opening. The punched-out flap is fixated on the tip of the catheter during retraction by vacuum suction through the catheter

We made, in each of the four cadaver heads, seven anastomoses on the main proximal cerebral arteries of the left hemisphere and seven anastomoses on the main proximal cerebral arteries of the right hemisphere. The anastomoses were sequentially constructed on: (1) the P1/BA tip, (2) the P2, (3) the ICA before its bifurcation, (4) the ICA bifurcation, (5) the A1, (6) the proximal M1, and (7) one of the M2 arteries. In two cadaver heads, ELANA anastomoses were created in the left hemisphere and SELANA anastomoses in the right hemisphere (Fig. 4). In the two other cadavers, the side of the anastomoses was reversed (Fig. 5). Attachment time intervals were measured from the first penetration of the recipient artery in the cadaver (by either microsuture or anastomosis device) until

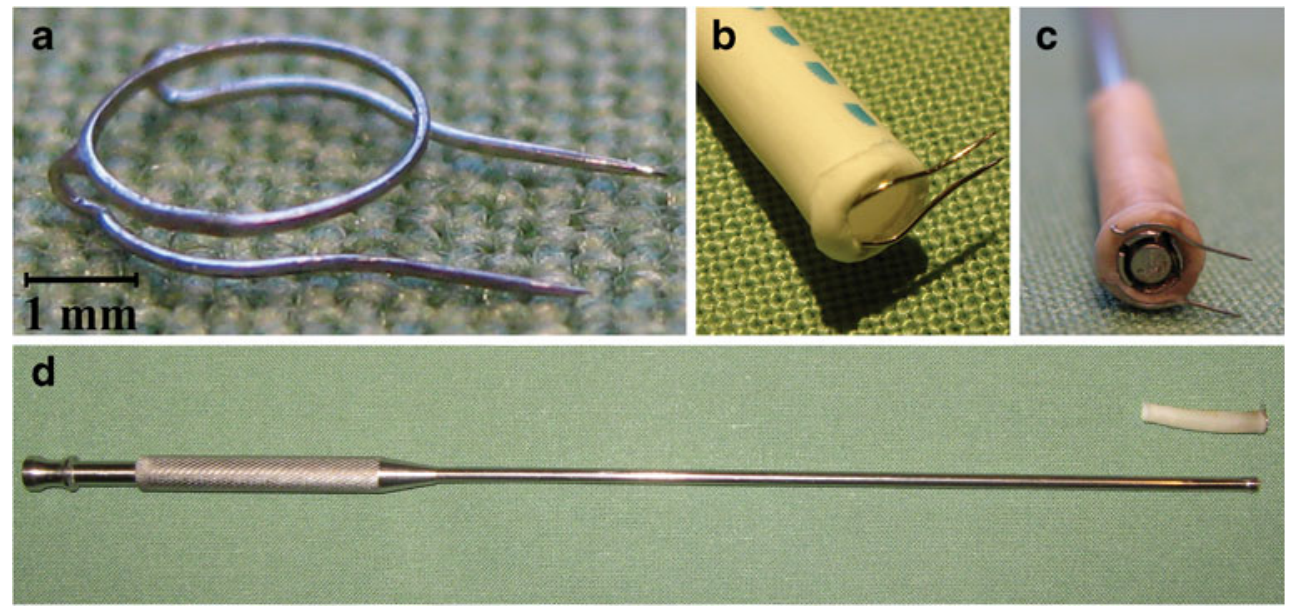

Fig. 2 Preparation of the SELANA anastomosis. a SELANA involves a ring with two pins. Each pin has a straight part and circumferential part. b The attachment of the ePTFE graft to the pins was performed by our technician, by bending and shaping the pins after puncturing the ePTFE graft from outside in and everting the graft over the ring. $\mathbf{c}$ and $\mathbf{d} \mathrm{A}$ specially designed applicator is introduced in the artificial graft until its tip is flush with the SELANA ring. The applicator contains an inner steel rod which can be pushed towards the tip with one click of the thumb on the upper part. This extends the diameter of the tip, fixating the applicator in the ring 


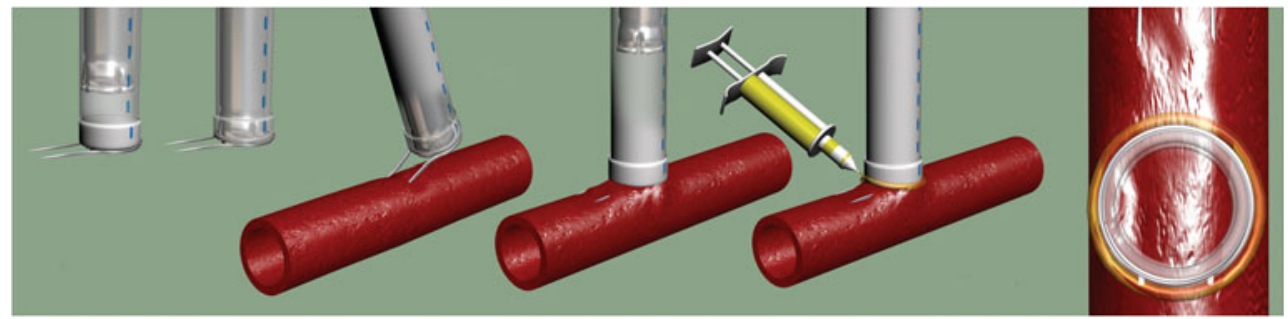

Fig. 3 Attachment of the SELANA anastomosis. The applicator is introduced in the ePTFE graft including the SELANA ring. The pins serve to attach the ePTFE graft to the recipient artery. First, both pins simultaneously puncture the artery wall outside in. The pins are then intra-luminally translated forward. If the longitudinal parts of the pins are completely in the vessel, resistance of the circumferential part is felt. The pins are then directed upward to puncture the recipient artery

the anastomosis was ready for lasing. The lasing procedure with the ELANA catheter was excluded from the time interval because it was the same in every anastomosis. This procedure consisted of introduction of the catheter in the bypass graft, 2 min of vacuum suction, two laser episodes $(10 \mathrm{~mJ}, 40 \mathrm{~Hz})$ of $5 \mathrm{~s}$, retraction of the catheter from the anastomosis and applying a clip over the bypass graft. After every anastomosis, the surrounding brain, all exposed cerebral arteries, and all exposed cranial nerves $(\mathrm{CN})$ were systematically inspected and scored for damage (yes-no) by an observer, other than the surgeon. If damage was observed, the video recordings were analyzed by two observers to find the exact cause of damage.

\section{Analysis}

To compare application time intervals between ELANA and SELANA, we used non-paired $t$ tests because in both groups,

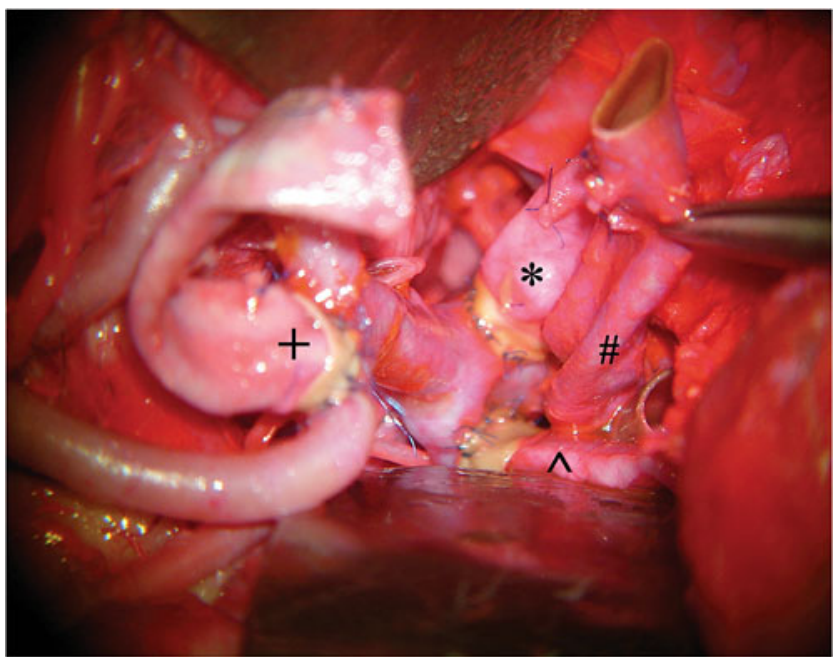

Fig. 4 Left side of cadaver 3. At this stage before lasing, four conventional ELANA anastomoses were attached (" proximal internal carotid artery (ICA), *distal ICA; ${ }^{+}$distal middle cerebral artery (M2); ${ }^{\wedge}$ proximal anterior cerebral artery (A1)) wall inside out. With a gentle second forward motion, the circumferential shape of each pin 'clicks' under the recipient artery wall. The straight part of each pin will be positioned outside the artery. The anastomosis is sealed with Bioglue (Cryolife ${ }^{\circledR}$ Inc., Kennesaw, GA, USA). Opening of the anastomosis is identical to the conventional ELANA anastomosis (Fig. 1b)

application time was normally distributed (KolgomorovSmirnov test, $P$ value $>0.05$ ). SPSS version 15.0 was used. Numbers are stated \pm standard deviation unless otherwise indicated.

\section{Results}

More ELANA anastomoses (26 of 28) than SELANA anastomoses (22 of 28 ) could be finished. However, more SELANA anastomoses (22 of the 28) than ELANA anastomoses (20 of 28 ) could be created without overt manipulation of surrounding structures.

During the creation of the 28 ELANA anastomoses, two anastomoses on the BA could not be finished because there was no space to apply a microsuture in minimally one quadrant. During the creation of the two other BA/P1 ELANA anastomoses, the CN III and perforators from the P1 and the BA tip had to be manipulated to create enough space to perform the anastomosis. During the construction

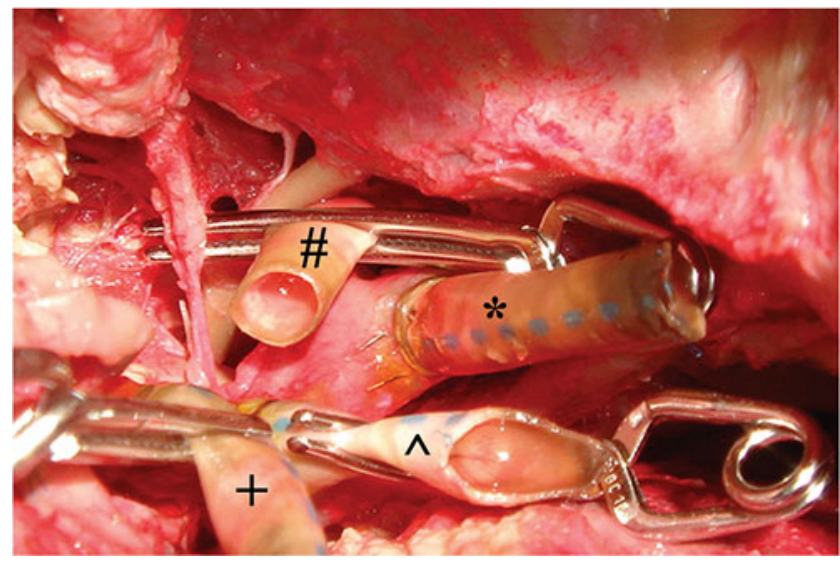

Fig. 5 Left side of cadaver 2. Four SELANA anastomoses (*proximal internal carotid artery (ICA) anastomosis; ${ }^{\wedge} \mathrm{ICA}$ bifurcation anastomosis; +middle cerebral artery anastomosis; and \#basilar artery anastomosis). The ICA was not lased open yet, the other three were successfully lased and temporary occluded with an aneurysm clip 
of two P2 ELANA anastomoses, a perforator from the posterior communicating artery ruptured and a hemoclip had to be placed on the artery. During two ELANA anastomoses on the ICA before the bifurcation, the CN II was manipulated several times by instrument shafts causing visible damage. After the construction of two M1 ELANA anastomoses, damage of frontal cerebral cortex was observed, also caused manipulation by instrument shafts.

During the creation of the 28 SELANA anastomoses, it appeared that the SELANA applicator was not suitable to apply anastomoses on spots which were not perpendicular or near perpendicular to the surgical corridor. Therefore, we could not apply SELANA anastomoses on all four A1 arteries, and not on two M1 arteries which were angulated sharply upward from the ICA bifurcation. During the application of the other 22 anastomoses, no complications, like rupture of the recipient arterial wall, occurred. Two of these anastomoses demanded slight manipulation of surrounding structures; during two BA anastomosis, the CNIII had to be mobilized and manipulated to create enough space.

At the anastomoses spots, were both the ELANA and SELANA technique could be performed (all except for the A1), the SELANA anastomosis showed to be significantly faster applicable than the ELANA anastomosis (Table 1). The mean time interval necessary to construct an ELANA anastomosis significantly increased with depth of the anastomosis. ELANA application time ranged from $12 \mathrm{~min}$ on the M2 anastomosis to $130 \mathrm{~min}$ on the P1/BA. With the SELANA anastomosis, the depth of the anastomosis was of significantly less influence to the application time. SELANA application time ranged from three minutes on the M2 to $11 \mathrm{~min}$ on the P1/BA (Table 1).

Flap retrieval rate by the ELANA catheter appeared to be not significantly different between the finished conventional ELANA anastomoses (20 of 26 flaps, $77 \%$ ) and the finished SELANA anastomoses (19 of 22 flaps, 86\%). Also, leakage of anastomoses after lasing was not significantly different between the ELANA anastomoses (six leakages, 23\%) and SELANA anastomoses (six leakages, 27\%).

\section{Discussion}

This feasibility study comparing the new experimental SELANA anastomosis with the conventional ELANA anastomosis in a pressurized cadaver model shows potential advantages of the SELANA anastomosis, considering its fast application and low complications. SELANA anastomoses could be simply applicated on deep anastomosis spots with minimal brain retraction, still benefiting of all the advantages of a non-occlusive technique.

However, the SELANA anastomosis design as described in this study still needs some improvement. At first, the anastomosis was not feasible on every intracranial anastomosis location. The special applicator should be redesigned to facilitate SELANA anastomoses on locations not perpendicular to the surgical corridor. Secondly, a surgical sealant is necessary to prevent the SELANA anastomosis in its current form from leaking after opening with the laser catheter. In this study, we used Bioglue ${ }^{\circledR}$, one of the rare sealants which adheres sufficiently to artificial grafts, but which is not FDA approved for intracranial use. Therefore, different dedicated vascular sealants in combination with the SELANA device should be tested in the future.

In this study, we used ePTFE grafts. There are multiple reasons to aim for a combination of SELANA with artificial grafts: (1) surgery to harvest the donor graft, with associated technical details and complications [8], is eliminated, (2) the SELANA ring can be preoperatively attached which reduces surgical time, and (3) the patient population is getting older, increasing the risk on low quality autologous vessels or even on the absence of the

Table 1 Number of completed anastomoses and application times

\begin{tabular}{|c|c|c|c|c|c|}
\hline Location & ELANA anastomoses & Application time (min) & SELANA anastomoses & Application time (min) & Mean difference \\
\hline M2 & 4 & $15 \pm 3$ & 4 & $4 \pm 1$ & $11(7-15)^{*}$ \\
\hline M1 & 4 & $24 \pm 8$ & 2 & $7 \pm 4$ & $17(5-29)^{*}$ \\
\hline $\mathrm{ICA}^{\mathrm{b}}$ & 8 & $45 \pm 6$ & 8 & $4 \pm 2$ & $41(33-49)^{*}$ \\
\hline A1 & 4 & $59 \pm 10$ & None & - & - \\
\hline $\mathrm{P} 2$ & 4 & $52 \pm 9$ & 4 & $5 \pm 3$ & $47(35-59)^{*}$ \\
\hline $\mathrm{P} 1 / \mathrm{BA}$ & 2 & $115 \pm 15$ & 4 & $8 \pm 3$ & $107(89-125)^{*}$ \\
\hline Total & 26 & & 22 & & \\
\hline
\end{tabular}

$M 2$ first generation middle cerebral artery branch, $M 1$ proximal middle cerebral artery, $I C A$ internal carotid artery, $A 1$ proximal anterior cerebral artery, $P 2$ posterior cerebral artery distal to posterior communicating artery, $P 1$ proximal posterior cerebral artery, $B A$ basilar artery

${ }^{\text {a }}$ Showing minutes $(95 \% \mathrm{CI})$

${ }^{\mathrm{b}}$ Four anastomoses were constructed on the ICA and four on the ICA bifurcation

$* P<0.05$ (unpaired $t$ test) 
vessels because of earlier cardiac or peripheral bypass surgery. In general, the 3-year patency of autologous grafts is only $74-83 \%$. [6, 9, 10]However, until now, artificial vessels are not proven to be as safe as autologous vessels. Substantial (pre)clinical research still has to be performed before a new generation of vascular substitutes will become clinically available. Therefore, in the first clinical application of the SELANA technique, we will use an autologous donor graft (the greater saphenous vein or the radial artery) in combination with the SELANA ring. Autologous donor grafts are still the golden standard for small diameter $(<3 \mathrm{~mm})$ vascular reconstructions. [4] The results of the current study would have been the same if these donor grafts were used because there is no difference in intracranial application mechanism.

A limitation of this study is the performance of all procedures by one surgeon (TPCVD). Although this facilitates comparison of variables between both study groups, it also introduces an observer bias. Therefore it might be dangerous to extrapolate the outcomes. However, differences between both tested anastomosis techniques were remarkably clear and significant. We therefore consider this study sufficient to show reliable differences between the ELANA and SELANA concept. At further preclinical testing, multiple other surgeons should evaluate the SELANA technique and compare it with the conventional ELANA technique.

A minor drawback of the cadaver model by Aboud et al. [1] was the stiffness of the arterial walls due to the preparation with formalin. Besides a different feel, this probably decreased the flap retrieval rate. Flap retrieval rate can be studied best in fresh arteries, so it is not useful to draw further conclusions from these data besides the comparison between the ELANA and SELANA subgroups. Despite the absence of coagulating blood, the anastomosis leakage in the model was limited because of the ideal viscosity of the artificial blood. The 'Aboud model' was easy to use and substantially reduced the use of laboratory animals for SELANA feasibility studies.

\section{Conclusion}

This comparative study shows potential advantages of the SELANA anastomosis over the ELANA anastomosis, considering its fast application and low complications. Further preclinical research should be performed in order to improve SELANA feasibility on angulated anastomosis spots. A logical next step is to assess long-term SELANA patency and endothelialization.

Acknowledgments The authors wish to thank R. Mansvelt-Beck for his technical support and R. Toelanie for his help in preparing Figs. 1 and 3.
Financial disclosure The equipment needed to create ELANA and SELANA anastomoses were provided free of charge by Elana bv, Utrecht, the Netherlands.

Bioglue ${ }^{\circledR}$ was provided free of charge for this study by Krijnen Medical Innovations bv, Beesd, The Netherlands.

Open Access This article is distributed under the terms of the Creative Commons Attribution Noncommercial License which permits any noncommercial use, distribution, and reproduction in any medium, provided the original author(s) and source are credited.

\section{References}

1. Aboud E, Al Mefty O, Yasargil MG (2002) New laboratory model for neurosurgical training that simulates live surgery. J Neurosurg 97(6):1367-1372

2. Heros RC, Lee SH (1993) The combined pterional/anterior temporal approach for aneurysms of the upper basilar complex: technical report. Neurosurgery 33(2):244-250

3. Krisht AF (2005) Transcavernous approach to diseases of the anterior upper third of the posterior fossa. Neurosurg Focus 19(2): E2

4. Nishibe T, Kondo Y, Muto A, Dardik A (2007) Optimal prosthetic graft design for small diameter vascular grafts. Vascular 15 (6):356-360

5. Nutik SL (1998) Pterional craniotomy via a transcavernous approach for the treatment of low-lying distal basilar artery aneurysms. J Neurosurg 89(6):921-926

6. Regli L, Piepgras DG, Hansen KK (1995) Late patency of long saphenous vein bypass grafts to the anterior and posterior cerebral circulation. J Neurosurg 83(5):806-811

7. Streefkerk HJ, Bremmer JP, van Weelden M, van Dijk RR, de Winter E, Beck RJ, Tulleken CA (2006) The excimer laser assisted nonocclusive anastomosis practice model: development and application of a tool for practicing microvascular anastomosis techniques. Neurosurgery 58(1 Suppl):ONS148ONS156

8. Sundt TM III, Sundt TM Jr (1987) Principles of preparation of vein bypass grafts to maximize patency. J Neurosurg 66(2):172180

9. van Doormaal TP, van der Zwan A, Verweij BH, Han KS, Langer DJ, Tulleken CA (2008) Treatment of giant middle cerebral artery aneurysms with a flow replacement bypass using the excimer laser assisted nonocclusive anastomosis technique. Neurosurgery 63 (1): $12-20$

10. van Doormaal TP, van der Zwan A, Verweij BH, Langer DJ, Tulleken CA (2008) Treatment of giant and large internal carotid artery aneurysms with a high-flow replacement bypass using the excimer laser assisted nonocclusive anastomosis technique. Neurosurgery 62(6 Suppl 3):1411-1418

11. Yasargil MG (1969) Microsurgery applied to neurosurgery. Georg Thieme, Stuttgart

\section{Comment}

There is an increased need for bypasses due to uncoilable and unclippable aneurysms or previously treated but growing remnants necessitating extraction from the circulation followed by subsequent revasculazation. Also, some skull-base tumors encircle major arteries which need to be sacrificed for complete tumor removal and then a high-flow bypass must be considered. We know already that dedicated 
neurovascular surgeons in high-volume centers should do the work and on regular basis. However, it still needs to be defined more accurately what kind of a bypass is sufficient in different situations, and ultimately which patients would benefit out of them.

This paper shows in cadavers how a possible improvement in an existing high-flow non-occlusive bypass technique could make it technically less demanding and time consuming. Our experience of the current ELANA technique has shown that it is very complex, demanding, and quite vulnerable to technical failures. Therefore, it often is impossible to predict intraoperative problems (e.g., in obtaining the flap or keeping the bypass patent) before surgery making the outcome unpredictable even in experienced hands. Therefore, there is still room for improvement in the ELANA technique. The SELANA technique seems to offer a simpler and faster solution instead of the often laborious suturing of the anastomoses in deep and narrow surgical corridors. It still needs to be tested thoroughly in large numbers of animals to see the risks of early thrombosis and arterial dissection with subsequent morbidity and mortality. With enthusiasm we await for further studies.

\section{Mika Niemelä}

Miikka Korja

Juha Hernesniemi

Helsinki, Finland

This paper describes a possible development of ELANA anastomotic technique in the living cadaver pressurized model developed at the Yasargil Microneurosurgery Laboratory of Little Rock. This interesting report is produce by a group that has gained major scientific consideration for experimental and clinic contributions in the field of laser assisted microsurgical anastomoses.

We know that the ELANA technique allows the execution of highflow non-occlusive bypasses in patients bearing complex aneurysms or skull base tumors requiring "flow replacement" for their surgical management. This work is proposed as proof of concept that in the experimental setting SELANA technique, which is characterized by a ring equipped with two pins pre-mounted on an artificial graft (ePTFE) with a diameter of $3 \mathrm{~mm}$, is feasible and somehow favorably comparable to the already established ELANA procedure. Although limited in the number of procedures, the experimental design is valid and sufficiently supporting the cautious conclusions of the authors. In general, both low- and high-flow anastomoses share the same critical issues; time to perform the procedure and risks of leakage from and vessel rupture at the anastomotic site, or stenosis of the recipient vessel. It seems that SELANA anastomoses are able to circumvent these problems. One of the problems encountered with this technique is performing the end-to-side suture on the receiving artery with eight microsutures, particularly technically demanding when dealing with deep vessels difficult to access.

Results of this study demonstrate that in this experimental setting the SELANA technique permits to carry out the anastomosis in a shorter time, especially on deep-seated vessels (P1/BA), with apparently less need for brain retraction. The disadvantages encountered essentially consist in the difficulties inherent in dealing with vessels not perpendicular to the surgical corridor and the need of applying a sealant to deal with post-lasering leakage.

In conclusion, the SELANA technique, taking into account the limitations inherent to the model selected for these experiments is a promising evolution of the conventional non-occlusive ELANA anastomosis. Further studies are to be carried out on the optimal type of donor graft. In fact, artificial vessels are not currently available in vascular neurosurgery, and preclinical studies on animal models from the same group (personal communication) utilize as a SELANA graft saphenous vein (on rabbit aorta) or common carotid artery (on porcine common carotid artery). I fully agree with the author's suggestion that other surgeons should evaluate the SELANA technique in the preclinical setting.

The authors are to be commended for the impact of their clinical and laboratory efforts on current and future neurovascular surgery.

Domenico d'Avella, Padova, Italy

Carlo Conti, Mestre, Italy 